\title{
Advanced methods of bacteriological identification in a clinical microbiology laboratory
}

\author{
Magdalena Elwira Żukowska ${ }^{1, A-B, D, F}$ \\ ${ }^{1}$ Chair and Department of Medical Microbiology, Medical University, Lublin, Poland \\ A - Research concept and design, B - Collection and/or assembly of data, C - Data analysis and interpretation, \\ $D$ - Writing the article, E-Critical revision of the article, F- Final approval of article
}

Żukowska ME. Advanced Methods of Bacteriological Identification in Clinical Microbiology Laboratory. J Pre-Clin Clin Res. 2021; 15(2): 68-72. doi: $10.26444 / j p c c r / 134646$

\begin{abstract}
Introduction and objective. Conventional, culture-based methods of bacterial identification and drug-susceptibility testing are considered the gold standard in medical microbiology. In recent years, classical microbiological methods have been supplemented with modern analytical and molecular methods. The aim of the review was to discusses the methods which have been permanently adapted to bacteriological microbiological diagnostics.

Abbreviated description of the state of knowledge. Currently, PCR, as well as other nucleic acid amplification tests and sequencing techniques, are part of the standard repertoire of microbiological diagnostics. With regard to the quality and speed of pathogen identification, the introduction of mass spectrometry techniques into routine microbiological diagnostics work-up has been revolutionary. Within a short time in many laboratories, Matrix-Assisted Laser Desorption/lonisation Time of Flight Mass Spectrometry (MALDI TOF MS) systems have almost completely replaced conventional biochemical pathogen identification.

Conclusions. Microbiological diagnostics is an indispensable element of a targeted therapy. The techniques used in the laboratory depend primarily on the laboratory's apparatus, the costs of the analysis, as well as the sensitivity and specificity of a method. However, regardless of the culture-based methods universality, advanced techniques have permanently established themselves in diagnostics. Confident information about the detected organism and treatment possibilities in a combination with the clinical context are conducive to successful therapy. Although modern methods still require validation and close collaboration between clinicians, microbiologists and bioinformaticians, these methods, once deemed to be the future, have already arrived.
\end{abstract}

\section{Key words}

Multiplex PCR, Nested PCR, Real-Time PCR, Next-Generation Sequencing, MALDI-MS

\section{INTRODUCTION}

Accurate pathogen identification is one of the basic tasks of a clinical microbiology laboratory, and is crucial for the microbiological diagnosis of infectious diseases and the selection of the most appropriate therapy. Therefore, diagnostic microbiology has been developing methods for rapid and accurate identification of invading microorganisms in clinical samples [1]. In recent years, classical microbiological methods, such as culture and identification, based on the phenotypic characteristics of the microorganisms, have been supplemented with modern analytical and molecular methods. Currently, PCR, as well as other NAAT and sequencing techniques are part of the standard repertoire of microbiological diagnostics. These procedures are widely used for the direct detection of pathogens or for the molecular identification of cultured microorganisms, for the recognition of resistance genes or virulence factors, and for genotype determination [2]. With regard to the quality and speed of pathogen identification, the introduction of mass spectrometry techniques into routine microbiological diagnostics work-up has been revolutionary. Within a

Address for correspondence: Magdalena Elwira Żukowska, Chair and Department of Medical Microbiology, Medical University, Chodźki 1, 20-093, Lublin, Poland

E-mail: magdalena.e.zukowska@gmail.com

Received: 10.02.2021; accepted: 18.03.2021; first published: 08.04.2021 short time in many laboratories, MALDI TOF MS systems have almost completely replaced conventional biochemical pathogen identification. Time has shown that these changes in microbiological diagnostics have significantly reduced the time needed to produce test results, and this has had an obvious clinical impact on the successful treatment of the patient [3].

Due to technological development, microbiological diagnostics is constantly expanding its range with new methods. The presented review discusses the methods which have been permanently adapted to bacteriological microbiological diagnostics. A selective literature search was conducted to collect current studies on the use of modern diagnostic methods in the microbiology laboratory.

\section{CONVENTIONAL DIAGNOSTICS METHODS}

Traditional, culture-based methods for the identification of microorganisms are considered the 'gold standard' with which other laboratory tests are compared [4]. These methods include Gram stain preparation, growing bacterial cultures on appropriate artificial media, and identification of bacteria on the basis of the biochemical profile individual for each species. Moreover, only a culture of bacteria in vitro allows determination of drug susceptibility and resistance 
phenotypes of the isolated pathogen. A great advantage of the conventional methods of microbiological diagnostics is that they are well-validated, and the obtained results are therefore reliable. Their affordability and availability make their presence obligatory in hospitals and private laboratories [5].

On the other hand, the identification of microorganisms with the use of traditional microbiology diagnostic methods have clear limitations due to their relatively low sensitivity and specificity to microorganisms that are nutritionally demanding, or whose biochemical activity is limited. Traditional methods can only be used in relation to the organisms with known growth requirements. The culturebased methods also do not allow the isolation of intracellular pathogens, as well as the differentiation of some very closelyrelated species. Moreover, the overall turnaround time for these tests is usually at least $48-72$ hours and, in addition, it takes weeks to detect slow-growing organisms. Such a delay in obtaining laboratory results may affect the treatment of serious infections. This is unacceptable if the diagnosis concerns a life-threatening condition [6-8].

\section{MODERN DIAGNOSTICS METHODS}

While the identification of microorganisms by culture is the gold standard in microbiology, the technique has some limitations which can be reduced by new diagnostic approaches, such as genotypic methods or methods based on the analysis of species-specific protein profiles. In recent years, these methods have proven useful in overcoming some of the limitations of traditional phenotypic procedures for detecting and characterising bacterial pathogens directly in clinical samples. Modern microbiology diagnostic methods are characteristic of the high sensitivity and specificity, and more importantly, a small amount of clinical material is needed to perform the analysis. Standard culture techniques generally require 48-72 hours to provide final results, whereas modern microbiology diagnostic methods have the potential to considerably reduce this turnaround time. Certainly, the solutions used in modern techniques allow bypassing the limitations encountered in phenotypic microbiological diagnostics. However, as a novelty they are still not fully validated. The complexity of the latest and most advanced methods and the interpretation of the obtained results require a close cooperation between clinicians, microbiologists and bioinformaticians. Only their close collaboration, combined with the use of computational tools, enables a critical assessment of the obtained result [7-9].

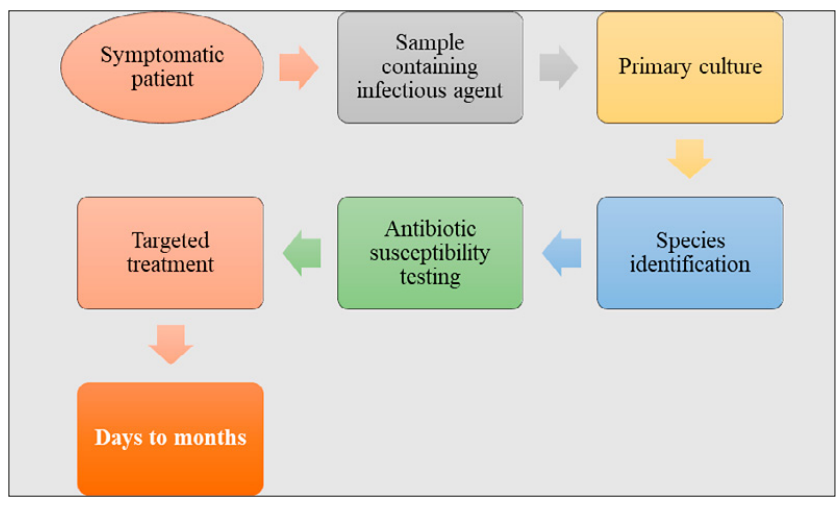

Figure 1. Conventional microbiology techniques

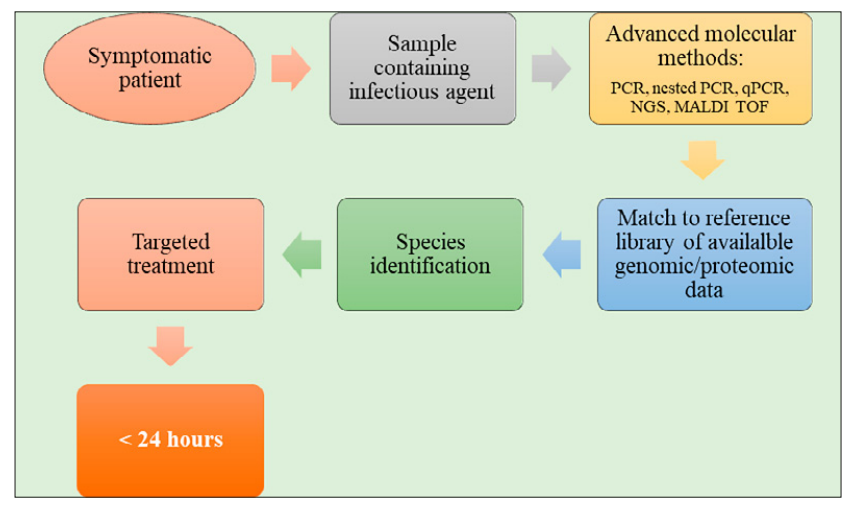

Figure 2. Advanced microbiology methods

\section{MOLECULAR METHODS}

Nucleic Acid Amplification Tests (NAATs). The development of the polymerase chain reaction (PCR) was a milestone in biological research, giving rise to the modern era of molecular diagnostics. With high sensitivity and specificity, NAATs provide new opportunities for the clinical laboratory in patient care and have become the new 'gold standard' in laboratory diagnosis of many infectious diseases [10].

PCR-based diagnostics have found application in a wide variety of microbes. Due to its high sensitivity, detecting from 1 - 100 genome equivalents of bacterial or fungal DNA, specificity and rate of amplification, PCR is recommended for the identification of organisms that cannot be cultured in vitro, or in cases where existing culture techniques are insensitive and / or require extended incubation times [11].

Currently, PCR and other NAAT techniques belong to the standard set of methods for microbiological diagnostics. They are widely used not only for the direct detection of pathogens in a clinical specimen, or for the molecular identification of cultured microorganisms, but also for the detection of resistance genes or virulence factors [12].

Although PCR is the most widely available nucleic acid amplification test, other strategies have also been developed, several of which have found clinical application [13]. The most frequently used PCR modifications in routine diagnostics are multiplex PCR, nested PCR and quantitative PCR (qPCR) [14]. In multiplex PCR, amplification of more than one target sequence in parallel is possible in a single PCR reaction by the incorporation of several pairs of primers specific for different bacterial or viral pathogens [11]. Therefore, multiplex PCR is regularly used to identify molecular species, especially in cases of patients presenting non-specific clinical symptoms which may result from infection of different pathogens or to examine associations of pathogens with the disease [14]. The ability to amplify several different DNA sequences in parallel significantly accelerates diagnostics and makes multiplex systems useful in routine laboratory diagnosis, enabling early initiation of potential treatment. Multiplex PCR has many applications and is broadly used in pathogen detection. In diagnostic microbiology it has been widely applied in the diagnosis of respiratory and genitourinary infections [9, $15,16]$.

Nested PCR is a modification of a PCR that was designed to improve sensitivity and specificity of the standard polymerase chain reaction technique. The method consists in performing a two-step amplification. The primary amplification reaction 
utilises a low specificity primer pair. This is followed by a reaction in which the obtained DNA serves as a template for the second cycle using primers of high specificity. This considerably increases sensitivity of the method without reducing its specificity, especially in case of suboptimal nucleic acid samples. Nested PCR has proven valuable for the detection of viral and bacterial pathogens when they are present in very low quantities. An example of the use of nested PCR is the detection of granulocytic ehrlichiosis in blood or Chlamydia pneumoniae in bronchoalveolar lavage (BAL) [15, $17,18]$. It is also possible to detect Mycobacterium tuberculosis in urine and blood samples $[19,20]$. A method employed by Cepheid in Xpert MTB/RIF tests not only allows for early tuberculosis diagnosis $(2 \mathrm{~h})$, but also indicates resistance of the strain to rifampicin (first line antituberculosis drug). The WHO, however, recommends caution and points to the need to confirm such a result with a microbial culture and a Ziehl-Neelsen stained sputum microscopic slide [21].

A significant advancement in PCR technology constitutes quantitative real-time PCR (qPCR) and its modifications that are faster than the traditional PCR, and do not require additional analysis of the obtained product as they run in a closed system [11]. While classic PCR detects the presence of foreign nucleic acids (viruses, bacteria, fungi and parasites), in the tested clinical sample, real-time PCR allows determination of the number of copies of the pathogen, which is possible due to fluorescent labelling of an amplified fragment. This technique is used in numerous virological tests. In bacteriology it is applied in the detection of Bordatella spp., Legionella spp., Mycoplasma, Ureaplasma and Chlamydia pneumoniae. Furthermore, qPCR facilitates the diagnostic process by detecting antibiotic resistance and encoding virulence factors genes. The undeniable benefits of this method are firstly, distinguishing contamination from the etiological factor, and secondly, assessing the stage of infection development. The duration of a test takes from 20 minutes to 3 hours, with sensitivity and specificity maintained on a high level.

One method of monitoring the production of amplicons in real time is to use non-specific fluorescent dyes (e.g., SYBR Green) which binds to double-stranded DNA produced during the amplification. However, this may generate signals giving false positive results. Higher accuracy is guaranteed with oligonucleotide hybridisation probes complementary to the amplified DNA fragment $[9,11,14-16]$.

High sensitivity and specificity, short duration and the possibility of automation in a closed system, are the attributes which bring NAATs to a microbiological laboratory. Their special role is played in the diagnosis of patients with lifethreatening conditions - sepsis, meningitis and encephalitis. A targeted therapy is highly desirable; therefore, the identification of an actual etiological factor in physiologically sterile fluids (i.e., blood and cerebrospinal fluid) is crucial [9]. However, the diagnosis of other systemic infections is associated with certain limitations that should be taken into account. Samples from physiologically non-sterile sites constitute a significant diagnostic challenge, not only due to the patient's natural microbiome which may interfere with the result. It is not always possible to determine whether the pathogen is an actual etiological factor or only colonises an infected system, especially when more than one pathogen is detected $[9,16]$.
Next Generation Sequencing (NGS). Originally described in 1976, the DNA sequencing method for which Frederick Sanger was awarded a second Nobel prize in his career, it was also a milestone in nucleic acid sequencing methods. Sanger sequencing precisely records a sequence of the nucleotides as they are arranged in an analyzed gene. Based on that, next-generation sequencing (NGS) has been employed in modern laboratories as an alternative [22, 23]. Currently, two main streams of NGS are mostly used: $16 \mathrm{~S}$ rRNA and shotgun metagenomics.

The $16 \mathrm{~S}$ ribosomal RNA is a component of the $30 \mathrm{~S}$ small subunit of the bacterial ribosome. It consists of 1,500 base pairs, the structure of which is divided into nine highly conserved and hypervariable regions (V1- V9). Conserved regions serve as a primer binding sites for the PCR reaction. The variable regions are useful for procaryotic identification purposes. 16S rRNA is used for very accurate species identification and is suitable for taxonomic purposes by comparing the analysed sequences with those from the database (such as SILVA, RDP, GreenGenes or NCBI) [23]. The greatest benefit of sequencing is the ability to detect even the most atypical microorganisms from a clinical specimen, which is helpful in the case of sepsis or meningitis, and can alsobe applied in the diagnosis of tuberculosis [21]. 16S rRNA sequencing enabled the analysis of the pulmonary system microbiome complexity [22]. It was also used to develop STI tests for Treponema pallidum, Mycoplasma genitalium, Mycoplasma hominis, Ureaplasma parvum and Ureaplasma urealyticum in genitourinary samples [23]. The method is limited by the databases which must already contain the recognised 16S rRNA sequence [24].

Shotgun metagenomics is a PCR-independent method; therefore, it does not require knowledge of the gene structure in order to design a primer binding site. It allows the sequencing of the whole organism's genome, which enables the discovery of new species. This is of particular importance in epidemiology, as it makes the development of rapid PCR diagnostic tests possible, e.g., in 2011 in Germany, the Shiga-toxigenic E. coli (STEC) O104:H4 strain caused the death of over 50 people [24]. It is also possible to use shotgun metagenomics in direct microbiological diagnostics, especially in highly challenging clinical cases of life-threatening infections of unknown etiology. NGS developed a new scientific research trend - analysis of the human microbiome. Analysis of the species comprised of the microbiome in various psychosomatic diseases, turns out to be helpful in diagnostics, treatment monitoring, determining a microorganism-host correlation, or excluding such a correlation $[14,24]$.

Sequencing remains an expensive method, the diagnostic value of which is limited by standardisation. NGS also requires software that enables the visualisation of the result, a database against which the result can be compared, and a bioinformatician who knows how to design the reaction and interpret the results, while excluding possible errors. The inability to standardise the method is a certain complication [24].

Currently, it is believed that the $16 \mathrm{~S}$ rRNA gene NGS method will be implemented sooner as a routine diagnostic method since it is faster, less complicated and cheaper, compared to shotgun metagenomics. To prevent biases and pitfalls of the 16S rRNA gene NGS method a 'MYcrobiota' platform has been developed, designed to overcome the 
greatest limitations of the method, avoid reduplication of errors and to share experience with other microbiologists, making it easier to use NGS in routine diagnostics [24].

Matrix-Assisted Laser Desorption/Ionisation - Time of Flight Mass Spectrometry (MALDI-TOF MS). The latest generation tool used in clinical microbiology to identify microorganisms is mass spectrometry - MALDI-TOF MS. The use of MS for pathogen identification was first proposed in the 1970s, but it was only later when the German biophysicists Franz Hillenkamp and Michael Karas developed the MALDITOF MS technique suitable for routine use [25]. MALDITOF uses mass spectrum analysis to list molecules in a sample assaying the mass-to-charge $(\mathrm{m} / \mathrm{z})$ ratio. It identifies and quantifies any ionised biological molecule with a mass in the range of $100 \mathrm{Da}-100 \mathrm{kDa}$. Matrix Assisted Laser Desorption and Ionisation (MALDI) is a phase in which a sample undergoes ionisation from the energy of a nitrogen laser beam. Excited molecules are transferred from the matrix to the analyte and cause desorption of the newlyionised allotments. The TOF technique measures the time of ions' flight using the fact that ions of different masses move at different speeds [7].

The major advantage of MALDI-TOF MS is the ability to identify microorganisms directly from body fluids (e.g., blood, cerebrospinal fluid), or urine. This is of special importance in life-threatening situations such as sepsis or meningitis [26]. Additionally, in the case of Enterobacterales, there is a possibility to detect carbapenemases, which indicates a treatment direction which can exclude betalactam antibiotics [27]. In other cases, this method can be used supplementarily, for example, in the diagnostics of bacterial diarrhea etiology. It shortens the diagnostic process by $2-3$ days, and only a few colonies are needed to run the analysis [26]. MALDI is also a valuable method in the case of Mycobacterium tuberculosis infection. An active disease can be diagnosed when CFP-10 and ESAT- 6 antigenic peptides secreted by virulent $M$. tuberculosis strains are detected in a blood sample [21].

In MALDI-TOF, identification of the microorganism is based on a comparison of a mass peaks which are specific for every species, with the patterns in the database. This unique protein profile is called a molecular 'fingerprint' [7, 25]. It ensures that the obtained result is highly specific, while the small amount of tested material needed to perform the analysis guarantees high sensitivity of the method. MALDITOF MS is rapid (less than one hour), relatively inexpensive, and a high throughput method $[3,26]$.

At present, however, the method is inappropriate for the differentiation of some closely related species (such as the Enterobacter cloacae group) and identification of encapsulated microorganisms (for instance Klebsiella pneumoniae or Haemophilus influenzae). The biggest limitation is that MALDI-TOF needs a bacterial culture in most cases. When the analysis is performed directly from clinical material, it demands the sample to be purified beforehand (e.g., from leukocytes by centrifugation) $[3,26]$. Also, it cannot provide information about the drug susceptibility profile; however, current research conducted on agar cultures with the addition of antibiotics gives hope for development in this direction in the future $[3,25,26]$.

\section{CONCLUSIONS}

Microbiological diagnostics is an indispensable element of a targeted therapy. The techniques used in the laboratory depend primarily on the laboratory's apparatus, costs of the analysis, as well as the sensitivity and specificity of a method. However, regardless of the culture-based methods universality, advanced techniques have permanently established themselves in diagnostics. High sensitivity and specificity, as well as their short duration, speak in favour of NAATs and MALDI-TOF MS. Additionally, almost unlimited horizons of identification with NGS application provides the opportunity for a proper diagnosis, even in the most unique clinical cases. The confident information about the detected organism and treatment possibilities in combination with the clinical context, are conducive to successful therapy. Although modern methods still require validation and close collaboration between clinicians, microbiologists and bioinformaticians, these method, once deemed to be the future, have already arrived.

\section{REFERENCES}

1.Schmidt TM. Encyclopedia of Microbiology. 4th ed. Academic Press, 2019.

2. Lau SKP, Teng JLL, Woo PCY. Bacterial Identification Based on Universal Gene Amplification and Sequencing. In: Advanced Techniques in Diagnostic Microbiology. Vol. 2: Applications. 3rd ed. Springer Nature Switzerland; 2018. p. 1-2.

3. Hou TY, Chiang-Ni C, Teng SH. Current status of MALDI-TOF mass spectrometry in clinical microbiology. J Food Drug Anal. 2019; 27(2): 404-414. doi: 10.1016/j.jfda.2019.01.001

4. Boyles TH, Wasserman S. Diagnosis of bacterial infection. South African Medical Journal 2015; 105(5): 419. doi: 10.7196/SAMJ.9647

5. Bunn TW, Sikarwar AS. Diagnostics: Conventional Versus Modern Methods, Journal of Advances in Medical and Pharmaceutical Sciences 2016, 8(4): 1-7. https://doi.org/10.9734/JAMPS/2016/25959

6. Szewczyk EM. Podłoża, próby i metody diagnostyczne. In: Szewczyk EM. Diagnostyka bakteriologiczna. 3rd edition. Warszawa: Wydawnictwo Naukowe PWN SA; 2019. p. 505-573.

7. Żabicka D, Literacka E. Nowoczesne metody wykrywania i identyfikacji bakterii. Forum Zakażeń 2013; 4(1): 65-72. doi: dx.doi.org/10.15374/ fz2013009

8. Kelley SO. New Technologies for Rapid Bacterial Identification and Antibiotic Resistance Profiling. SLAS Technol. 2017; 22(2): 113-121. doi: $10.1177 / 2211068216680207$

9. Vila J, Gómez MD, Salavert M, et al. Métodos de diagnóstico rápido en microbiología clínica: necesidades clínicas. Enferm Infecc Microbiol Clin. 2017; 35(1): 41-46. doi: 10.1016/j.eimce.2017.01.014

10. Valones MA, Guimarães RL, Brandão LA, et al. Principles and applications of polymerase chain reaction in medical diagnostic fields: a review. Braz J Microbiol. 2009; 40(1): 1-11. doi: 10.1590/S151783822009000100001

11. Yang S, Rothman R. PCR-based diagnostics for infectious disease uses, limitations, and future applications in acute-care settings. Lancet Infect Dis. 2004; 4(6): 337-348. doi: 10.1016/S1473-3099(04)01044-8

12. Idelevich E, Reischl U, Becker K. New microbiological techniques in diagnosis of bloodstream infections. Dtsch Arztebl Int. 2018; 115(49): 822-832. doi: 10.3238/arztebl.2018.0822

13. Persing DH, Tenover FC, Hayden RT, et al. Molecular Microbiology: Diagnostic Principles and Practice. 3rd edition. 2016.

14. Buchan BW, Ledeboer NA. Emerging technologies for the clinical microbiology laboratory. Clin Microbiol Rev. 2014; 27(4): 783-822. doi: 10.1128/CMR.00003-14

15. Szemraj M. Metody molekularne w diagnostyce mikrobiologicznej. In: Szewczyk EM. Diagnostyka bakteriologiczna. 3rd edition. Warszawa: Wydawnictwo Naukowe PWN SA; 2019. p. 475-490.

16. Stratton CW. Tang YW. Interpretation and Relevance of Advanced Technique Results. In: Advanced Techniques in Diagnostic Microbiology. Vol. 2: Applications. 3rd edition. Springer Nature Switzerland; 2018. p. 711-740. 
17. Massung RF, Slater K, Owens JH, et al. Nested PCR assay for detection of granulocytic ehrlichiae. J Clin Microbiol. 1998, 36(4): 1090-1095. doi: 10.1128/JCM.36.4.1090-1095.1998

18. Tondella ML, Talkington DF, Holloway BP, et al. Development and Evaluation of Real-Time PCR-Based Fluorescence Assays for Detection of Chlamydia pneumoniae. J Clin Microbiol. 2002; 40(2): 575-583. doi: 10.1128/JCM.40.2.575-583.2002

19. Cruz HL, et al. Evaluation of a Nested-Pcr for Mycobacterium Tuberculosis Detection in Blood and Urine Samples. Braz. J. Microbiol. 2011; 42(1): 321-329. doi: 10.1590/S1517-83822011000100041

20. Figueirêdo da Costa Lima J, de Moraes Rêgo Guedes G, Falcão de Araújo Lima J, et al. Single-tube nested PCR assay with in-house DNA extraction for Mycobacterium tuberculosis detection in blood and urine. Revista da Sociedade Brasileira de Medicina Tropical. 2015; 48(6): 731-738. http://dx.doi.org/10.1590/0037-8682-0210-2015

21. Alves da Silva D, de Pina LC, Rêgo AM, et al. Advances in the Diagnosis of Mycobacterium tuberculosis Infection. In: Advanced Techniques in Diagnostic Microbiology. Vol. 2: Applications. 3rd edition. Springer Nature Switzerland; 2018. p. 101-136.

22. Morganti S, Tarantino P, Ferraro E, et al. Next Generation Sequencing (NGS): A Revolutionary Technology in Pharmacogenomics and Personalized Medicine in Cancer. Adv Exp Med Biol. 2019; 1168: 9-30. doi: 10.1007/978-3-030-24100-1_2
23. Boers SA, Jansen R, Hays JP. Understanding and overcoming the pitfalls and biases of next-generation sequencing (NGS) methods for use in the routine clinical microbiological diagnostic laboratory. Eur J Clin Microbiol Infect Dis. 2019; 38(6): 1059-1070. doi: 10.1007/s10096-01903520-3

24. Muldrew KL. Molecular Diagnostics of Sexually Transmitted Diseases: Bacterial, Trichomonas, and Herpes Simplex Virus Infections. In: Advanced Techniques in Diagnostic Microbiology. Vol. 2: Applications. 3rd edition. Springer Nature Switzerland; 2018. p. 67-100.

25. Hosseini S, Martinez-Chapa SO. Principles and mechanism od MALDIToF-MS analysis. In: Hosseini S, Martinez-Chapa SO. SpringerBriefs in Applied Sciences and Technology; 2017. p. 1-19. doi: 10.1007/978981-10-2356-9_1

26. Singhal N, Kumar M, Kanaujia PK, et al. MALDI-TOF mass spectrometry: an emerging technology for microbial identification and diagnosis. Front Microbiol. 2015; 6: 791. https://doi.org/10.3389/ fmicb.2015.00791

27. Niu S, Chen L. Molecular Detection and Characterization of Carbapenem-Resistant Enterobacteriaceae. In: Advanced Techniques in Diagnostic Microbiology. Vol. 2: Applications. 3rd edition. Springer Nature Switzerland; 2018. p. 165-186. 